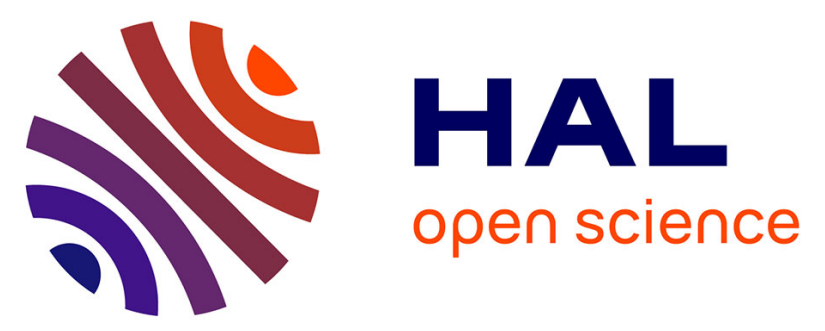

\title{
Nouveaux milieux, nouveaux gibiers, nouveaux chasseurs? Évolution des pratiques cynégétiques dans les Pyrénées du Tardiglaciaire au début du Postglaciaire
}

Sandrine Costamagno, David Cochard, Jean-Georges Ferrie, Véronique Laroulandie, Nathalie Cazals, Mathieu Langlais, Nicolas Valdeyron, Morgane Dachary, Michel Barbaza, Didier Galop, et al.

\section{To cite this version:}

Sandrine Costamagno, David Cochard, Jean-Georges Ferrie, Véronique Laroulandie, Nathalie Cazals, et al.. Nouveaux milieux, nouveaux gibiers, nouveaux chasseurs? Évolution des pratiques cynégétiques dans les Pyrénées du Tardiglaciaire au début du Postglaciaire. Bulletin de la Société préhistorique française, 2008, 105 (1), pp.17-27. halshs-00965573

\section{HAL Id: halshs-00965573 \\ https://shs.hal.science/halshs-00965573}

Submitted on 25 Mar 2014

HAL is a multi-disciplinary open access archive for the deposit and dissemination of scientific research documents, whether they are published or not. The documents may come from teaching and research institutions in France or abroad, or from public or private research centers.
L'archive ouverte pluridisciplinaire HAL, est destinée au dépôt et à la diffusion de documents scientifiques de niveau recherche, publiés ou non, émanant des établissements d'enseignement et de recherche français ou étrangers, des laboratoires publics ou privés. 


\title{
Sandrine COSTAMAGNO, David COCHARD, Jean-Georges FERRIÉ, Véronique LAROULANDIE, \\ Nathalie CAZALS, Mathieu LANGLAIS, Nicolas VALDEYRON, Morgane DACHARY, Michel BARBAZA, \\ Didier GALOP, Hélène MARTIN et Sylvie PHILIBERT
}

\begin{abstract}
Résumé
Les changements climatiques survenus durant le Tardiglaciaire et au début du Postglaciaire ont modifié de manière considérable l'environnement naturel des populations préhistoriques. Pour certains auteurs, ces modifications seraient à l'origine d'une intégration massive des petits animaux dans l'alimentation. Dans les Pyrénées françaises et sur leurs marges, une intensification de la capture des petits gibiers apparaît à la fin du Dryas ancien. Les données archéozoologiques couplées aux données paléoenvironnementales montrent que le réchauffement et les modifications environnementales qui en découlent ne sont pas directement responsables des changements observés dans l'exploitation du milieu animal. En raison de la multiplicité des facteurs déclenchants et de leur interaction probable, il est cependant difficile, dans l'état actuel des recherches, d'appréhender les causes qui ont conduit les populations du Tardiglaciaire à intégrer les petits gibiers dans leur économie et leur univers symbolique.
\end{abstract}

\begin{abstract}
The climatic changes which occurred during the Late Glacial and at the beginning of the Post Glacial considerably modified the natural environment of prehistoric populations. For certain authors, these modifications were at the origin of a massive integration of small animals in the diet. In the French Pyrenees and on their borders, broadening diets occur at the end of the Older Dryas. Archaeozoological data coupled with palaeoenvironmental data show that the warmer climate and environmental modifications were not directly responsible for the changes observed in the exploitation of animals. Because of the multiplicity of the triggering factors and their probable interaction, it is however difficult, in the current state of research, to apprehend the causes which led the Late Glacial populations to integrate small game in their economy and their symbolic system.
\end{abstract}

Les changements climatiques survenus durant le Tardiglaciaire et au début du Postglaciaire ont modifié de manière considérable l'environnement naturel dans lequel vivaient les populations préhistoriques. Le réchauffement et l'augmentation des précipitations, outre une modification décisive du couvert végétal, ont 
progressivement libéré des territoires jusque-là peu propices à l'implantation humaine (Jalut et al., 1992; Heinz et Barbaza, 1998). Dans le Sud de la France, les vastes étendues steppiques parcourues par de grands troupeaux d'Ongulés sont alors peu à peu remplacées par des forêts, peuplées d'une faune moins grégaire et plus compartimentée. Par ailleurs, l'augmentation des températures s'accompagne d'un accroissement de la durée de la saison végétative des plantes qui, dès lors, sont susceptibles d'être plus intensément exploitées par les groupes humains. De telles modifications environnementales sont probablement à l'origine de changements adaptatifs majeurs de la part des groupes de chasseurs-cueilleurs tant du point de vue de l'exploitation et de la gestion des ressources alimentaires que des modalités d'occupation des territoires (Barbaza, 1999).

Dans le cadre de ce travail, nous essayons d'évaluer si, dans les Pyrénées françaises et ses marges, le réchauffement est effectivement à l'origine de l'apparition de nouvelles stratégies alimentaires, en rupture avec les économies de chasse paléolithiques. Sur le plan chronoculturel, l'étude s'est limitée aux gisements du Tardiglaciaire et du début de l'Holocène (1800010000 cal. BP) (Reimer et al., 2004). Cette période voit se succéder, dans les Pyrénées, au moins trois grandes cultures distinctes (le Magdalénien, l'Azilien et le Sauveterrien) correspondant aux trois grands cycles évolutifs (Paléolithique, Épipaléolithique et Mésolithique) traditionnellement identifiés. Dans le détail cependant, des phénomènes de contemporanéité et de chevauchements partiels ne sont pas à exclure et le paysage culturel peut être éventuellement plus complexe que ne le laisse penser la précédente énumération (existence de faciès aziloïdes différents de l'Azilien s. s., notamment).

\section{L'INTÉGRATION MASSIVE DES PETITS ANIMAUX DANS L'ALIMENTATION : HYPOTHËSES ENVIRONNEMENTALES}

Selon certains auteurs, les bouleversements climatiques intervenus à la fin du Tardiglaciaire seraient responsables de l'intégration dans les régimes alimentaires des préhistoriques de nouvelles ressources, aboutissant à un élargissement de la diète des chasseurs-cueilleurs (Rowley-Conwy, 1986). Ce postulat repose sur le modèle de l'amplitude de la diète (diet breadth model), lui-même fondé sur le principe d'optimisation (Winterhalder, 1981). Selon ce modèle, la sélection des ressources alimentaires est guidée par la contrainte coût/bénéfice, et ce afin d'obtenir le meilleur rendement énergétique possible. De façon générale, les petits gibiers ne sont pas des ressources à forte rentabilité pour l'Homme du fait de leur taille réduite et de leur grande mobilité. Des bouleversements climatiques, comme le réchauffement holocène, initié dès le Tardiglaciaire, peuvent être à l'origine de l'intégration de ces gibiers de faible rang selon deux cas de figures :
1. Une abondance exceptionnelle de ces ressources et une relative stabilité des proies de plus haut rangs.

2. Une forte diminution de toutes les ressources de rang supérieur (Bridault, 1993).

Dans le premier cas, la biomasse disponible sur le territoire est augmentée, ce qui procure au chasseurcueilleur un environnement plus sûr, lui permettant de surmonter d'éventuelles périodes de stress alimentaire. En outre, le choix de l'acquisition résulte de la seule augmentation de l'efficience de la proie, son abondance provoquant la réduction de son coût de recherche. Dans le deuxième cas, la situation est tout autre puisque la biomasse diminue, ce qui crée un stress environnemental. Pour le surmonter et faire face à la pénurie des ressources de haut rang, le groupe humain va être contraint d'exploiter les ressources à faible rentabilité disponibles sur son territoire. Il s'agit donc d'un modèle «misérabiliste» en opposition au premier modèle dit «d'abondance».

\section{LA GRANDE FAUNE CHASSÉE}

À la fin du Tardiglaciaire, une recomposition des cortèges fauniques d'Ongulés est observée à l'échelle du territoire français (Delpech, 1987; Bignon, 2003; Bridault et Fontana, 2003). Or, selon le modèle de l'amplitude de la diète, les Ongulés étant des gibiers de plus haut rang que les proies de petite taille, toute modification de leurs cortèges peut avoir des répercussions sur le degré d'intégration des petites espèces dans l'alimentation. Une évaluation de la transformation des spectres fauniques dans les Pyrénées et de ses conséquences sur les stratégies de chasse mises en œuvre est donc un préalable pour identifier les facteurs responsables d'une éventuelle exploitation accrue des petits gibiers au cours du Tardiglaciaire.

\section{Une grande faune en mutation}

Les Ongulés sont largement dépendants de la végétation, elle-même étant étroitement liée au climat (Delpech et al., 1983). La composition des spectres fauniques sur un gisement donné peut donc nous renseigner, une fois intégrées les questions de sélection, sur les conditions climatiques de l'époque à laquelle l'assemblage osseux s'est constitué. De telles reconstitutions paléoenvironnementales reposent sur le principe d'actualisme qui postule que les exigences écologiques d'un animal sont constantes dans le temps. Selon divers travaux (Delpech et al., 1983; Griggo, 1996), quatre groupes d'Ongulés peuvent être définis dans la zone géographique considérée :

- le groupe arctique : Renne;

- le groupe de montagne : Bouquetin, Chamois;

- le groupe forestier: Cerf, Chevreuil, Sanglier, Aurochs ;

- le groupe de steppe continentale : Cheval, Bison. 
À l'exception de la grotte de Bourrouilla, dominée par le Cerf (Fosse, 1999; Costamagno, 2006), la première phase du Tardiglaciaire (Dryas ancien et début du Bölling) se caractérise par l'abondance des Ongulés de steppe continentale et/ou arctiques, le groupe de montagne pouvant être particulièrement fréquent sur certains gisements (fig. 1 et tabl. 1). C'est durant l'Alleröd, aux alentours de 14000 cal. BP, que le groupe forestier devient prépondérant dans les spectres de chasse. Ainsi, dans la couche 7 de Troubat et le niveau F6 de Rhodes II, datés respectivement de 11520 $\pm 100 \mathrm{BP}-$ Ly 913 (13623-13200 cal. BP1) et 12100 \pm 150 BP - MC 997 (14593-13647 cal. BP), on observe une nette augmentation du groupe de milieu boisé, qui, pour la première fois, comprend non seulement le Cerf mais également le Chevreuil et le Sanglier, signe d'une nette amélioration climatique. Localement, certaines données peuvent cependant apparaître en discordance avec ce mouvement général. Ainsi, et contrairement à d'autres régions françaises où le Renne disparaît un peu après $14000 \mathrm{cal}$. BP (Bridault et al., 2000), le Magdalénien final de Bourrouilla (Costamagno, in Dachary et al., 2004), comme les niveaux aziliens de Duruthy (Delpech, 1978) et Dufaure (Altuna et Mariezkurrena, 1995), pourraient témoigner d'une disparition tardive de cette espèce dans la partie occidentale de la chaîne. Selon F. Delpech (ibid.), ce décalage pourrait s'expliquer par la proximité des reliefs montagneux pyrénéens, qui aurait favorisé la constitution de zones refuges propices à sa survie ${ }^{2}$.

\section{Des spectres fauniques diversifiés : une constante du Magdalénien au Sauveterrien}

La recomposition des cortèges fauniques observée à la fin du Tardiglaciaire est marquée par une diminution puis une disparition des grands troupeaux d'Ongulés (chevaux, rennes) au profit d'espèces plus solitaires (cerfs, sangliers, chevreuils). Les premiers, pouvant être abattus en masse lors de grandes chasses collectives, offrent parfois un rang tellement élevé par rapport aux autres Ongulés disponibles dans l'environnement que, dans certaines régions (Bassin parisien, Périgord), tout au moins à certaines saisons, ils ont été les seuls gibiers exploités par les groupes paléolithiques (Enloe, 1999; Costamagno, 2003). Au contraire, les Ongulés de milieu fermé, en raison de leurs mœurs solitaires, possèdent des rangs équivalents, ce qui doit aboutir, selon le principe d'optimisation, à des spectres de chasse plus diversifiés (Costamagno et Mateos Cachorro, sous presse).

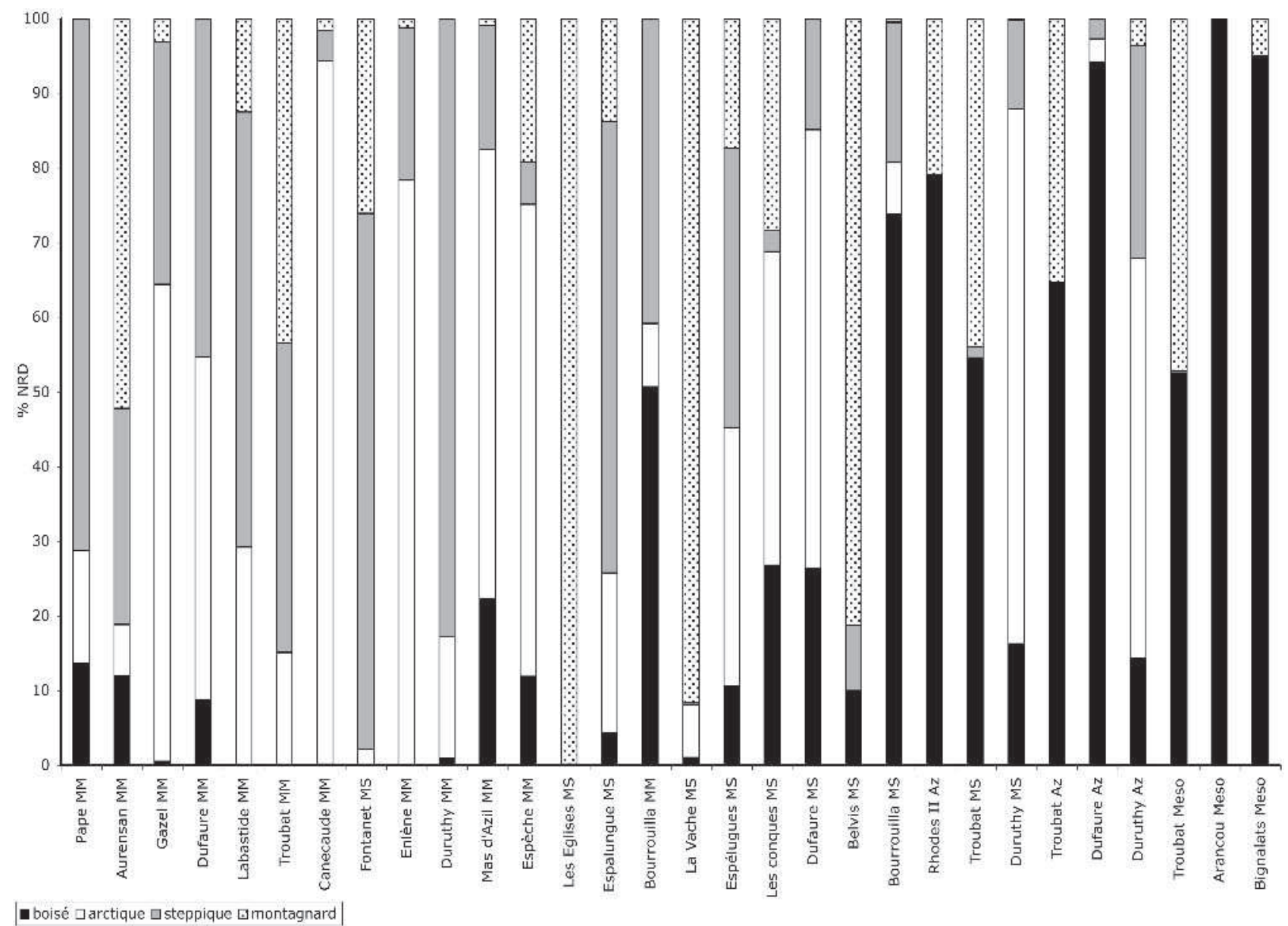

Fig. 1 - Fréquence relative des groupes écologiques d'Ongulés au sein des ensembles osseux pyrénéens classés par ordre chronologique (MM : Magdalénien moyen; MS : Magdalénien supérieur; AZ : Azilien; Meso : Mésolithique). 


\begin{tabular}{|l|l|l|l|}
\hline \multicolumn{1}{|c|}{ Gisement } & Département & \multicolumn{1}{c|}{ Culture } & \multicolumn{1}{c|}{ Références bibliographiques } \\
\hline Aurensan & H.-Pyr. & Magdalénien & Clot, 1971 et 1972; Clot et Omnès, 1979 \\
\hline Belvis & Aude & Magdalénien & Fontana, 1998b \\
\hline Bignalats & Pyr.-A. & Mésolithique & Altuna et Marsan, 1986 \\
\hline Bourrouilla & Pyr.-A. & Magdalénien & Fosse, 1999; Costamagno, in Dachary et al., 2004 \\
\hline Canecaude & Aude & Magdalénien & Fontana, 1998a \\
\hline Conques (les) & Pyr.-O. & Magdalénien & Moigne et al., 1998; Baills, 2003 \\
\hline Dufaure (abri) & Landes & Magdalénien, Azilien & Altuna et Mariezkurrena, 1995 \\
\hline Duruthy & Landes & Magdalénien, Azilien & Delpech, 1978 \\
\hline Églises (les) & Ariège & Magdalénien & Delpech et Le Gall, 1983; Delpech et Villa, 1993 \\
\hline Enlène & Ariège & Magdalénien & Delpech, 1981 \\
\hline Espalungue & Pyr.-A. & Magdalénien & Clot, 1986 \\
\hline Espèche & H.-Pyr. & Magdalénien & Clot et Omnès, 1979; Clot, 1985 \\
\hline Espélugues & H.-Pyr. & Magdalénien & Clot et Omnès, 1979; Clot, 1985 \\
\hline Fontanet & Ariège & Magdalénien & Clottes, 1979 \\
\hline Gazel (grotte de) & Aude & Magdalénien, Azilien & Fontana, 1998a \\
\hline Labastide & H.-Pyr. & Magdalénien & Clot, in Omnès, 1982 \\
\hline Mas d'Azil & Ariège & Magdalénien & Patou, 1984 et 1985 \\
\hline Pape (grotte du) & Landes & Magdalénien & Patou-Mathis et Boukhima, 1996 \\
\hline Rhodes II & Ariège & Magdalénien, Azilien & Delpech, 1983 \\
\hline Troubat & H.-Pyr. & Magdalénien, Azilien, Mésolithique & Martin, 1994; Costamagno, 2005 \\
\hline Vache (la) & Ariège & Magdalénien & Pailhaugue, 1995 \\
\hline
\end{tabular}

Tabl. 1 - Sites pris en compte dans l’étude (H.-Pyr. : Hautes-Pyrénées, Pyr.-A. : Pyrénées- Atlantiques, Pyr.-O. : Pyrénées-Orientales).

La diversité des spectres fauniques chassés a été documentée grâce à l'indice de Shannon ${ }^{3}$ (Grayson, 1984) : plus l'indice est fort, plus le spectre est diversifié; des valeurs proches de zéro indiquent des ensembles osseux monospécifiques, c'est-à-dire spécialisés. Cette diversité apparaît comme une constante tout au long de la période considérée (fig. 2). Il faut toutefois signaler que les spectres ultraspécialisés (indice de Shannon $<0,3$ ) ne sont documentés qu'au Magdalénien sur certaines espèces (rennes et bouquetins) qui ne sont, par ailleurs, pas forcément grégaires.

\section{L'EXPLOITATION DE LA PETITE FAUNE}

Dans cette synthèse, le terme "petite faune » se réfère aux oiseaux et aux mammifères dont le poids à l'âge adulte est compris entre $500 \mathrm{~g}$ (Putois) et $15 \mathrm{~kg}$ (Blaireau).

\section{Une intensification de la chasse aux oiseaux à la fin du Dryas ancien}

Bien que présents dans la plupart des gisements répertoriés, la fréquence de l'ensemble de ces petits gibiers excède rarement $10 \%$ des restes osseux déterminés (Costamagno et Laroulandie, 2004). La part effective dans la subsistance des groupes humains est vraisemblablement plus faible, en raison de la diversité potentielle des agents d'accumulation de cette petite faune (Homme, carnivores, rapaces...). Sur les gisements ayant fait l'objet d'analyses archéozoologiques,

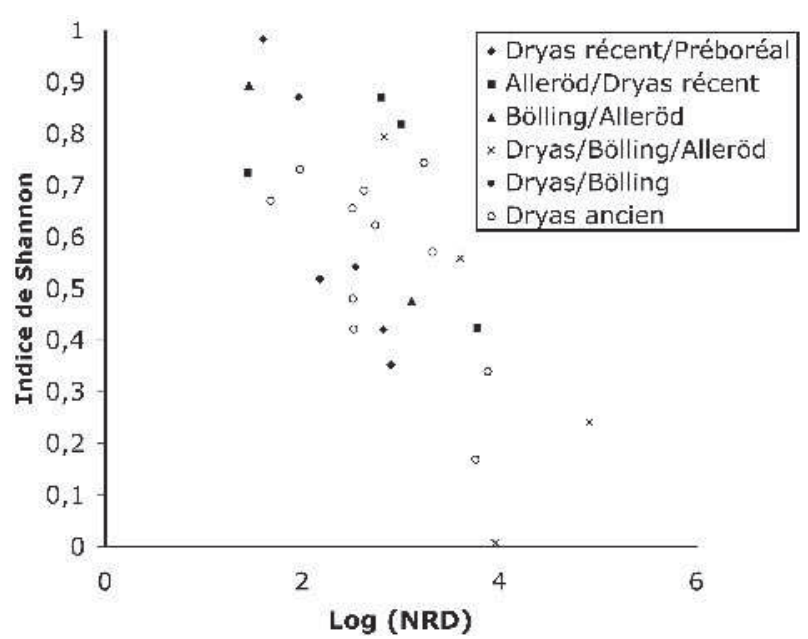

Fig. 2 - Indice de diversité des spectres fauniques d'Ongulés dans les gisements magdaléniens, aziliens et sauveterriens des Pyrénées françaises.

la gamme des petits mammifères exploités apparaît variée tout au long de la période (Lièvre, Renard, Blaireau, Chat forestier...). Au Dryas ancien, seule la grotte Gazel (Aude) atteste d'une exploitation importante du Lièvre variable 4 . Parmi les oiseaux, les Lagopèdes, la Chouette harfang et, dans une moindre mesure, le Chocard sont les espèces chassées du Dryas ancien à la fin du Bölling. Á l'échelle du Sud de la France, ces choix relèvent d'une tendance générale à l'intensification de l'exploitation des petits animaux qui commence à se manifester vers $16000 \mathrm{cal}$. BP. Le site de la Vache, avec ses 50000 ossements de Lagopèdes représentant près de 5000 individus, témoigne 
de ce nouvel intérêt (Laroulandie, 2005). À la fin du Tardiglaciaire, le spectre de chasse se modifie et incorpore de nouvelles espèces comme le Pigeon (Vilette, 1999).

\section{Une exploitation des petits gibiers à la fois alimentaire, technique et symbolique}

Le faible nombre d'études archéozoologiques disponibles limite nos connaissances sur les modalités d'exploitation des petits gibiers. Il se dégage néanmoins une diversité dans les techniques de boucherie mises en œuvre et dans les sous-produits recherchés (viande, moelle, peau, plume...).

La consommation de la viande est attestée pour l'ensemble des petits animaux chassés pour l'ensemble de la période étudiée. Sur le site magdalénien de la Vache, l'abondance des stries de boucherie correspond à un prélèvement quasi systématique de la viande des lagopèdes et des chocards (Laroulandie, 2005). En revanche, aux Églises, site voisin et subcontemporain, la faible fréquence de traces de découpe sur les carcasses de lagopèdes témoigne de pratiques bouchères distinctes (Laroulandie, 2004). Ainsi que le suggèrent les données contextuelles relatives à l'acquisition des matières premières siliceuses (Clottes et Simonnet, 1979; Simonnet, 1998), cette différence pourrait refléter des besoins et des traditions techniques de deux groupes distincts. Pour les Léporidés, parallèlement à l'exploitation de la viande, l'extraction de la moelle apparaît comme une pratique récurrente (Cochard, 2004).

$\mathrm{Au}$ delà de l'aspect alimentaire, les petits gibiers participent à l'enrichissement de la gamme des matières premières exploitées. Ainsi, les Magdaléniens ont utilisé les fourrures de lièvres variables (Fontana, 2003a) ainsi que leurs incisives comme éléments de parure sur le site de Gazel (Sacchi 2003), les os de chouettes harfang comme matrice à aiguilles (Chauvière et Eastham, in Dachary et al., 2005 et 2006) ainsi que ses griffes sur les gisements de Dufaure et de Bourrouilla (Eastham, 1998). L'utilisation des plumes pour les empennages ou comme éléments de décor est probable mais n'apparaît pas clairement documentée à partir du seul registre archéologique. Au Mas d'Azil, une matrice d'extraction d'aiguilles sur un tibia de Lièvre $^{5}$ ainsi que de nombreux os longs d'oiseaux décorés sont signalés dans les niveaux magdaléniens (Chollot, 1964).

\section{DISCUSSION}

\section{L'influence des facteurs environnementaux sur l'acquisition des ressources animales}

D'après les études palynologiques, le développement de la forêt sur le versant nord de la chaîne pyrénéenne s'amorce un peu avant 15000 cal. BP (Bölling) au sein d'un paysage qui reste encore largement ouvert (Jalut et al., 1998). L'amélioration climatique enregistrée au cours de l'Alleröd (14000-12900 cal. BP) se traduit par une augmentation du couvert forestier et une recomposition effective des cortèges fauniques avec l'avènement des espèces de milieu boisé. Dans cet environnement, la biomasse d'Ongulés potentiellement exploitable est inférieure à celle disponible dans les milieux ouverts (Ramade, 1984, cité dans Delpech, 1999). Selon l'hypothèse «misérabiliste» (cf. supra), cette diminution des proies de haut rang induirait une augmentation de l'exploitation des petits gibiers (ressource de faible rang). Or, dans les Pyrénées, l'importance de ces proies reste relative puisque, lorsqu'elles sont exploitées, elles fournissent au maximum $5 \%$ du poids des ressources carnées (Costamagno et Laroulandie, comm. orale). La minutie apportée à la préparation culinaire des oiseaux, notamment sur le site de la Vache, dénote un fort investissement, réduisant d'autant la rentabilité de ces ressources. En outre, l'incorporation au régime alimentaire d'oiseaux mais également de poissons (Le Gall, 2003) s'intensifierait vers 16000 cal. BP alors même que les grands troupeaux d'Ongulés de milieu ouvert sont toujours abondants.

Selon l'hypothèse «d'abondance», l'intensification de la capture de petits gibiers serait liée à une augmentation exceptionnelle de leurs effectifs au sein de l'environnement. Dans ce cadre, les rassemblements saisonniers des lagopèdes, des saumons ou des lièvres sont susceptibles de faciliter leur capture. Mais dans l'état actuel des recherches, aucun facteur environnemental ne semble pouvoir expliquer le développement de ces rassemblements autour de et après $16000 \mathrm{BP}$.

Même si les cortèges fauniques de grands Ongulés se modifient largement au cours de la période considérée, la mise en œuvre de chasses sur des proies diversifiées d'herbivores apparaît comme une constante. Ponctuellement, certains gisements magdaléniens témoignent d'une chasse spécialisée sur un seul gibier. Bien que non documenté dans les Pyrénées françaises, ce type de gisement ciblé sur l'acquisition d'un herbivore particulier est également connu durant le Mésolithique, comme à la Balma Margineda (Andorre) dominée par le Bouquetin (Geddes et al., 1985 et 1990) ou dans d'autres régions, notamment le Massif central et le Sud-Est de la France où plusieurs gisements se caractérisent par une chasse quasi exclusive de l'Aurochs (Pasty et al., 2002 ; Fontana, 2003b; Helmer et Monchot, 2006).

Ainsi, au cours de la phase chronologique qui nous intéresse, les Ongulés font le plus fréquemment l'objet de chasses diversifiées, ce qui dénote des stratégies d'acquisition relativement similaires. Par ailleurs, l'intensification de la capture des petits gibiers apparaît dès la fin du Dryas ancien. Dans les Pyrénées, en dehors des poissons, cette intensification ne semble pas perdurer à l'Épipaléolithique. Cette désaffection contraste avec les observations faites dans le nord de l'Aquitaine où le Lapin devient, à cette époque, l'espèce dominante dans plusieurs gisements du Quercy et de la Dordogne (Séronie-Vivien, 1994; Cochard, 2004; Cochard et Brugal, 2004). Les 
informations sur le Mésolithique sont limitées du fait du nombre réduit d'études archéozoologiques. Toutefois, les activités halieutiques paraissent en nette régression (Le Gall, 1993) tandis que l'intérêt pour les mollusques terrestres augmente (Barbaza, 1999; Lubell, 2004).

\section{Le rôle des facteurs socioculturels dans l'exploitation des petits gibiers}

Si l'ensemble des données disponibles, en montrant notamment qu'il n'y a pas de synchronisme strict dans les évolutions, permet de réfuter l'idée d'une action directe des changements climatiques sur l'exploitation des ressources carnées, quelles peuvent être les causes de ces modifications? Plusieurs hypothèses pouvant expliquer les changements enregistrés dans la composition des archéofaunes de la fin du Tardiglacaire et du début du Postglaciaire ont été proposées dans d'autres régions du globe (voir bilan dans Cochard, 2004). Dans l'état actuel de nos recherches, il est difficile de tester leur validité dans les Pyrénées mais elles offrent cependant une base de réflexion dans nos perspectives de recherche.

Les Léporidés, les rongeurs et certains oiseaux résistent mieux que les Ongulés à une forte prédation en raison de leurs taux élevés de reproduction et de fertilité (Stiner et al., 1999). Une fréquence importante de petits animaux dans une archéofaune témoignerait donc, selon cette hypothèse, d'un accroissement de la pression cynégétique induit par une réduction de la mobilité (Aura Tortosa et al., 2002) ou une expansion démographique (Stiner, 2001).

Les capacités techniques des sociétés humaines ont également une influence sur le degré d'efficience d'une ressource (Holliday, 1998). L'apparition et/ou l'optimisation d'une arme peut permettre la diminution des coûts de poursuite et de capture d'une proie donnée, augmentant ainsi son rang d'efficience.

Un autre facteur à considérer est l'organisation sociale du travail selon le sexe et l'âge des individus. Le regard de l'ethnologie sur ce point paraît particulièrement instructif car il souligne, chez certains groupes de chasseurs-cueilleurs, le rôle primordial des femmes et des enfants dans la capture des petits gibiers (e. $g$. Bird et Bliege Bird, 2000). L'existence d'un large spectre de chasse incluant la petite faune pourrait donc refléter un changement dans la répartition des activités au sein du groupe.

Pour J. Haws et B. Hockett (2004), l'avantage reproductif ne se situe pas dans l'optimisation énergétique des ressources mais dans la maximisation des éléments nutritifs essentiels (protéines, graisse, carbohydrates, vitamines, eau et minéraux) afin de diminuer notamment les risques de mortalité fœtale. Dans ce cadre, l'acquisition des petits gibiers serait donc susceptible d'enrichir la gamme des éléments nutritifs essentiels consommés.

Pour l'ensemble de ces hypothèses, la finalité de l'acquisition d'une ressource par un prédateur est uniquement d'ordre alimentaire. Ce préalable paraît réducteur quand il est transféré aux sociétés humaines car elles ont également des demandes en matières premières (Chaix, 1988; Fontana, 2003a; Müller, 2004). Les facteurs socioculturels jouent dans ce cadre un rôle prédominant. En plus d'être fonctionnels, la fourrure, les dents, les coquilles et les plumes sont autant d'éléments ornementaux et/ou symboliques potentiels. L'exploitation de la petite faune pourrait ainsi être liée à une volonté d'affirmation sociale au sein de sociétés en évolution, marquées par des processus d'affichage individuels ou collectifs en augmentation.

\section{Les modes d'exploitation des ressources biotiques trouvent-ils écho dans ceux des ressources minérales?}

De nombreuses analyses technologiques des industries lithiques sont maintenant disponibles dans l'espace pyrénéen et permettent d'esquisser une comparaison dans les modalités d'exploitation et d'utilisation des ressources biotiques et minérales. En outre, les ressources minérales sont d'excellents traceurs géographiques, la connaissance des gîtes d'approvisionnement et la détermination pétrographique des roches taillées permettant de mettre en rapport gîtes de matière première et sites archéologiques. Enfin, la relative stabilité dans le temps et l'espace des ressources minérales permet de s'affranchir, au moins en théorie, de la variable paléoenvironnementale, du moins pour tout ce qui concerne la disponibilité des ressources ${ }^{6}$, les changements environnementaux pouvant influer évidemment sur les besoins et les pratiques. Cette approche nécessite une bonne connaissance à la fois des séries lithiques régionales mais aussi des ressources disponibles dans la zone d'étude considérée. Des recherches sur les ressources minérales entreprises sur le long terme (Briois et al., 1999; Simonnet, 1999; Tarriño, 2001), des études régionales centrées sur l'analyse de séries archéologiques (e. g. Lacombe, 1998; Cazals, 2000; Dachary, 2002) et des travaux collectifs récents sur l'ensemble de la zone pyrénéenne et les Cantabres (Cazals et al., 2005) permettent aujourd'hui de documenter avec précision les ressources et les productions.

Dans les Pyrénées, qui sont globalement dépourvues de matériaux adaptés au débitage de grandes lames, c'est au cours du Magdalénien que l'on constate la plus grande circulation de matériaux entre le massif et les aires géographiques limitrophes, en particulier le Bassin aquitain (Lacombe, 1998; Simonnet, 1999; Séronie-Vivien, 2002). Cette circulation s'organise sous des formes diversifiées : volumes à débiter entiers comme réserve de matière première, outils finis aux fonctions diversifiées... Cette organisation est en partie liée à un outillage sur lames dont les dimensions et les techniques d'obtention sont contraignantes et qui nécessite des matières premières à la fois de bonne qualité et de grandes dimensions (Simmonet, 1982; Lacombe, 1998 ; Cazals, sous presse). Par conséquent, 
lorsque les Magdaléniens vont dans des zones à faible potentiel, comme les Pyrénées centrales, ils transportent avec eux un stock prévisionnel de matériaux allochtones qui complète l'exploitation sur place des silex locaux. Dans certains contextes, des schémas opératoires qui s'adaptent à des blocs de petites dimensions peuvent être mis en œuvre (Dachary, 2002).

La transformation progressive des assemblages lithiques à la fin du Tardiglaciaire s'observe, notamment, dans la dimension et la normalisation des outillages qui diminuent et dans les savoir-faire techniques mis en œuvre, moins contraignants vis-à-vis de l'approvisionnement en matière première. Cette perte d'exigence autorise l'exploitation accrue des matériaux disponibles dans l'environnement immédiat de l'habitat. Comme il a été montré dans d'autres régions (Valentin, 2005), le passage du Magdalénien à l'Azilien s'opère graduellement et la véritable rupture techno-économique a lieu ensuite, avec les groupes de l'Épipaléolithique et du Mésolithique qui produisent des outillages de moins en moins investis techniquement. Les chaînes opératoires apparaissent également moins fractionnées dans l'espace (Valdeyron, 2000).

Comment corréler ces observations aux données sur la faune? Plusieurs remarques, qui seront autant de pistes à explorer dans l'avenir, peuvent être formulées. Si l'on se fie à l'origine des matériaux lithiques retrouvés dans les sites du piémont pyrénéen, les territoires parcourus durant le Magdalénien sont très vastes, incluant notamment les grandes plaines parcourues par les grands troupeaux d'Ongulés. À la fin de cette période et au début de l'Holocène, l'environnement proche semble au contraire valorisé par la collecte, au détriment de stratégies plus complexes d'acquisition et de transport.

En même temps, il est légitime de s'interroger sur les acteurs. Si les données de la faune et de l'ethnologie laissent supposer un changement dans la répartition des activités au sein du groupe, qu'en est-il des équipements lithiques? L'existence d'une hiérarchie sociale des compétences techniques au Magdalénien, telle qu'elle est a été démontrée sur le site d'Étiolles U5, dans le Bassin parisien (Pigeot, 1987), et suggérée par les grandes lames du Magdalénien moyen pyrénéen (Simonnet, 1982), semble disparaître à la fin du Magdalénien. À travers la moindre place économique accordée à la lame dans l'outillage des technocomplexes postérieurs, l'hypothèse d'un changement socio-économique pourrait également être envisagée.

Par ailleurs, au cours du Tardiglaciaire et plus encore au début du Postglaciaire, la microlithisation croissante des armatures est le signe, généralement présenté comme incontestable, d'une systématisation de l'usage de l'arc et de la flèche, généralisation favorisée pour partie tout au moins par les changements environnementaux. La forte dispersion des ressources pourrait également être responsable de l'émergence de nouvelles formes d'organisation des groupes, caractérisées par une diminution de leur taille, la famille réduite devenant vraisemblablement la cellule de base. La taille des gisements, qui est en constante diminution à partir de la fin du Magdalénien, illustre bien ce processus (Barbaza, 1981; Rozoy, 1978 et 1992; Valdeyron, 1994). On peut alors imaginer que l'augmentation de la chasse des petits gibiers, lorsqu'elle est avérée, signe également la transformation de l'organisation sociale et économique des groupes, incluant une réduction de leur taille («familles»), voire une redistribution des rôles hommes-femmes, celles-ci voyant augmenter leur part dans l'acquisition des ressources alimentaires, tant par le biais de la capture de petites proies que par la collecte ou la cueillette, vraisemblablement très rentables, des produits végétaux.

\section{CONCLUSION}

$\mathrm{Au}$ vu des données disponibles, l'exploitation alimentaire des petites proies qui s'amorce dans les Pyrénées dès la fin du Dryas ancien n'apparaît pas comme une conséquence directe des changements environnementaux. Cette modification, qui trouve notamment un parallèle dans la gestion et la confection des équipements lithiques, participe aux mutations socioéconomiques marquant cette période. Les liens et les interactions qui unissent les composantes du système dans lequel s'inscrit l'évolution de ces cultures passées restent à expliciter dans leur complexité. La poursuite des travaux selon une approche systémique ainsi qu'une meilleure résolution du cadre chronologique sont pour cela nécessaire.

Remerciements : Ces recherches ont pu être menées grâce à une subvention de recherche octroyée par la fondation Fyssen.

\section{NOTES}

(1) Oxcal v. 4.0 : Bronk Ramsey, 1995 et 2001 ; Intcal04 : Reimer et al., 2004.

(2) Un programme de datations radiocarbone sur des os de rennes provenant de niveaux de la fin du Tardiglaciaire est en cours afin de vérifier la validité de l'hypothèse d'une perduration tardive du Renne au sein de la chaîne pyrénéenne (Costamagno, fondation Fyssen).

(3) $-\sum \mathrm{p}_{\mathrm{i}} \ln \mathrm{p}_{\mathrm{i}} / \ln \mathrm{S}$ avec $\mathrm{pi}=\mathrm{NRDi} / \mathrm{NRD}$, NRDi est le nombre de restes déterminés d'une espèce donnée, NRD est le nombre de restes déterminés total et $\mathrm{S}$ est le nombre de taxons présents dans l'assemblage

(4) Ce gisement a livré plus de 8000 restes de Lièvre qui représentent près de $50 \%$ des restes osseux identifiés.

(5) Cette pièce a été décrite comme un os d'oiseau (cf. Laroulandie, 2000).

(6) L'évolution de l'environnement végétal comme celle des couvertures superficielles (en particulier les accumulations périglaciaires de bas de versants) ont pu jouer sur l'accessibilité aux ressources minérales, comme l'ont montré A. Turq et ses collaborateurs (2000) pour le Turonien du Fumélois dont les affleurements sont recouverts au début du Mésolithique. 


\section{RÉFÉRENCES BIBLIOGRAPHIQUES}

ALTUNA J., MARIEZKURRENA K. (1995) - Les restes osseux de macromammifères, in L.G. Straus dir., Les derniers chasseurs de rennes du monde pyrénéen. L'abri Dufaure: un gisement tardiglaciaire en Gascogne, Mémoire de la Société Préhistorique française, t. XXII, Paris, p. 181-211.

ALTUNA J., MARSAN G. (1986) - Le gisement préhistorique de la grotte du Bignalats à Arudy (Pyrénées-Atlantiques). Première partie : présentation des fouilles et étude de la faune de mammifères, Archéologie des Pyrénées occidentales, t. 6, p. 53-73.

AURA TORTOSA J. E., VILLAVERDE BONILLA V., PEREZ RIPOLL M., MARTINEZ VALLE R., GUILLEM-CALATAYUD P. M (2002) - Big game and small prey: Palaeolithic and Epipalaeolithic economy from Valencia (Spain), Journal of Archaeological Method and Theory, vol. 9, p. 215-268.

BAILLS H. dir. (2003) - Les Conques : des chasseurs magdaléniens et leur territoire, ERAUL, t. 101, Liège, 222 p.

BARBAZA M. (1981) - Recherches sur l'Épipaléolithique en Languedoc et Catalogne, thèse de $3^{\mathrm{e}}$ cycle, École des hautes Études en Sciences sociales, Toulouse, 480 p., 105 fig.

BARBAZA M. (1999) - Les civilisations postglaciaires - La vie dans la grande forêt tempérée, La Maison des Roches, Paris, $126 \mathrm{p}$

BIGNON O. (2003) - Diversité et exploitation des Équidés au Tardiglaciaire en Europe occidentale. Implications pour les stratégies de subsistance et les modes de vie au Magdalénien et à l'Azilien ancien du Bassin parisien, thèse de doctorat, université Paris X - Nanterre, $836 \mathrm{p}$.

BIRD D.W., BLIEGE BIRD R. (2000) - The ethnoarchaeology of juvenile foragers: shellfishing strategies among Meriam children, Journal of Anthropological Archaeology, vol. 19, p. 461-476.

BRIDAULT A. (1993) - Les économies de chasse épipaléolithiques et mésolithiques dans le Nord de la France, thèse de doctorat, université Paris X, Paris, 552 p.

BRIDAULT A., FONTANA L. (2003) - Enregistrement des variations environnementales par les faunes chassées, dans les zones de moyenne montagne d'Europe occidentale, au Tardiglaciaire et au début de l'Holocène, in M. Patou-Mathis et $\mathrm{H}$. Bocherens dir., Le rôle de l'environnement dans les comportements des chasseurs-cueilleurs préhistoriques, BAR International Series, 1105, Oxford, p. 55-65.

BRIDAULT A., CHAIX L., PION G., OBERLIN C., THIÉBAULT S., ARGANT J. (2000) - Position chronologique du Renne (Rangifer tarandus L.) à la fin du Tardiglaciaire dans les Alpes du Nord françaises et le Jura méridional, Le Paléolithique supérieur récent nouvelles données sur le peuplement et l'environnement, Actes de la table ronde de Chambéry, 12-13 mars 1999, Mémoire de la Société préhistorique française, t. XXVIII, Paris, p. 47-57.

BRIOIS F., CHALARD P., GRÉGOIRE S, LACOMBE S. SERVELLE C., SIMONNET R. (1999) - Lithothèque des matières premières siliceuses, Bilan scientifique de la région Midi-Pyrénées 1998, Direction régionale des Affaires culturelles Midi-Pyrénées, service régional de l'Archéologie, Toulouse, p. 242-243.

BRONK RAMSEY C. (1995) - Radiocarbon calibration and analysis of stratigraphy: The OxCal program, Radiocarbon, vol. 37, p. 425-430.

BRONK RAMSEY C. (2001) - Development of the radiocarbon calibration program OxCal., Radiocarbon, vol. 43, p. 355-363.

CAZALS N. (2000) - Constantes et variations des traits techniques et économiques entre le Magdalénien inférieur et moyen : analyse de productions lithiques du nord de la Péninsule ibérique, thèse d'université, université de Paris I, Paris, 590 p.

CAZALS N. (sous presse) - Quelles relations de part et d'autre des Pyrénées à l'époque magdalénienne?, in N. Cazals et X. Terradas dir., Frontières naturelles et frontières culturelles dans les Pyrénées préhistoriques, Actes du colloque de Tarascon-sur-Ariège, mars 2004 Museo de Barcelona, Ministerio de la Cultura de Cataluña, Barcelone.
CAZALS N., BAENA J., BARTROLI R., BON F., BRACCO J.-P., CLEMENTE I., FUERTES PRIETO N., GONZALEZ URQUIJO J. E., IBAÑEZ J. J., MAILLO FERNANDEZ J., MANGADO LLACH X., ORTEGA i COBOS D., ORTEGA I., TERRADAS X. (2005) - Des faciès et des hommes : réflexions sur les productions d'éclats au Paléolithique supérieur dans les Pyrénées françaises et espagnoles, in J. Jaubert et M. Barbaza dir., Territoires, déplacements, mobilité, échanges, Actes du $126^{e}$ congrès national des Sociétés historiques et scientifiques, Toulouse, avril 2001, CTHS, Paris, p. 161172.

CHAIX L. (1988) - L'alimentation carnée dans le Mésolithique alpin : choix ou nécessité ?, Anthropozoologica, t. 2, p. 27-32.

CHOLLOT M. (1964) - Musée des Antiquités nationales - Collection Piette - Art mobilier préhistorique, édition des Musées nationaux, Paris

CLOT A. (1971) - Recherches d'Édouard Lartet sur la paléontologie quaternaire et la préhistoire des Hautes-Pyrénées, Société archéologique et historique du Gers, p. 394-400.

CLOT A. (1972) - Révision de quelques ossements de la collection Philippe et synthèse sur les gisements pléistocènes de Baudéan, Elysée-Cottin et Aurensan supérieur près de Bagnères-de-Bigorre, Bulletin de la Société Ramond, p. 39-64.

CLOT A. (1985) - Déterminations de paléontologie quaternaire dans le bassin de l'Adour (deuxième série, Pyrénées-Atlantiques), Archéologie des Pyrénées occidentales, t. 5, p. 205-222.

CLOT A. (1986) - Déterminations de paléontologie quaternaire dans le bassin de l'Adour (troisième série, Pyrénées-Atlantiques), Archéologie des Pyrénées occidentales, t. 6, p. 141-166.

CLOT A., OMNÈS J. (1979) - Premiers datages radiocarbone du Magdalénien des Hautes-Pyrénées, Bulletin de la Société préhistorique française, t. 76, p. 324-339.

CLOTTES J. (1979) - Ornolac-Ussat-les-Bains - Grotte de Fontanet, Gallia Préhistoire, t. 22, p. 653-654.

CLOTTES J., SIMONNET R. (1979) - Le Paléolithique final dans le bassin de Tarascon-sur-Ariège, d'après les gisements des Églises (Ussat) et de Rhodes II (Arignac), in D. de Sonneville-Bordes dir., La fin des temps glaciaires en Europe, chronostratigraphie et écologie des cultures du Paléolithique final, Colloques internationaux du CNRS $n^{\circ} 271$, Talence, 1977, CNRS, Paris, p. 647-659.

COCHARD D. (2004) - Les Léporidés dans la subsistance des Paléolithiques du Sud de la France, thèse de doctorat, université de Bordeaux I, Bordeaux, 346 p.

COCHARD D., BRUGAL J.-P. (2004) - Importance des fonctions de sites dans les accumulations paléolithiques de Léporidés, in J.-P. Brugal et J. Desse dir., Petits animaux et sociétés humaines, $X X I V^{\text {s }}$ rencontres internationales d'Archéologie et d'Histoire d'Antibes, octobre 2003, APDCA-CNRS, Antibes, p. 283-296.

COSTAMAGNO S. (2003) - Exploitation de la grande faune au Magdalénien dans le Sud de la France, in S. Costamagno et V. Laroulandie dir., Mode de vie au Magdalénien : les apports de l'archéozoologie, Actes du XIV congrès UISPP, Liège, septembre 2001, BAR International Series, 1144, Oxford, p. 73-88.

COSTAMAGNO S. (2005) - Mobilité, territoires de chasse et ressources animales au Magdalénien final en contexte pyrénéen : le niveau 7a de la grotte-abri du Moulin (Troubat, Hautes-Pyrénées), in J. Jaubert et M. Barbaza dir., Territoires, déplacements, mobilité, échanges, Actes du $126^{e}$ congrès national des Sociétés historiques et scientifiques, Toulouse, avril 2001, CTHS, Paris, p. 371-383.

COSTAMAGNO S. (2006) - Archéozoologie des grands mammifères des gisements de la falaise du Pastou, in M. Dachary dir., Les Mag daléniens à Duruthy, catalogue de l'exposition «Les Magdaléniens à Duruthy », Imprimerie Rochelaise, La Rochelle, p. 20-29.

COSTAMAGNO S., LAROULANDIE V. (2004) - L'exploitation des petits vertébrés dans les Pyrénées françaises du Paléolithique au 
Mésolithique : un inventaire taphonomique et archéozoologique, in J.-P. Brugal et J. Desse dir., Petits animaux et sociétés humaines $X X I V^{\text {s }}$ rencontres internationales d'Archéologie et d'Histoire d'Antibes, octobre 2003, APDCA-CNRS, Antibes, p. 403-416.

COSTAMAGNO S., MATEOS CACHORRO A. (sous presse) - Milieu animal de part et d'autre de la chaîne pyrénéenne : implications sur les modes de subsistance au Magdalénien, in N. Cazals et X. Terradas dir., Frontières naturelles et frontières culturelles dans les Pyrénées préhistoriques, Actes du colloque de Tarascon-sur-Ariège, mars 2004, Museo de Barcelona, Ministerio de la Cultura de Cataluña., Barcelone.

DACHARY M. (2002) - Le Magdalénien des Pyrénées occidentales, thèse de doctorat nouveau régime, université de Paris X, t. $1: 299$ p., 47 fig., t. 2 : XLVII pl.

DACHARY M., CHAUVIÈRE F.-X., COSTAMAGNO S., DAULNY L., DETRAIN L., FERRIER C. (2004) - La grotte de Bourrouilla (Pyrénées atlantiques), DFS de fouille programmée, service régional de l'Archéologie d'Aquitaine, 200 p.

DACHARY M., CHAUVIÈRE F.-X., COSTAMAGNO S., DAULNY L., EASTHAM A., FERRIER C., LE GALL O. (2005) - La grotte de Bourrouilla (commune d'Arancou, Pyrénées-Atlantiques, 64). DFS de la fouille programmée 2005, exemplaire multigraphié, service régional de l'Archéologie d'Aquitaine, $230 \mathrm{p}$.

DACHARY M., CHAUVIÈRE F.-X., DAULNY L., EASTHAM A., FERRIER C. (2006) - Grotte de Bourrouilla. Commune d'Arancou, Pyrénées-Atlantiques (64). DFS de la fouille programmée 2006, exemplaire multigraphié, service régional de l'Archéologie d'Aquitaine, $130 \mathrm{p}$.

DELPECH F. (1978) - Les faunes magdaléniennes et aziliennes du gisement de Duruthy, à Sorde-l'Abbaye (Landes), in R. Arambourou dir., Le gisement préhistorique de Duruthy à Sorde-l'Abbaye (Landes). Bilan des recherches de 1958 à 1975, Mémoire de la Société préhistorique française, t. XIII, Paris, p. 110-116.

DELPECH F. (1981) - La faune magdalénienne de la salle des Morts à Enlène, Montesquieu-Avantès (Ariège), in J. Clottes dir., La Préhistoire du Quercy dans le contexte de Midi-Pyrénées, Compte rendu de la XXI ${ }^{e}$ session, congrès préhistorique de France, Société préhistorique française, CNRS, Paris, p. 65-69.

DELPECH F. (1983) - Les faunes du Paléolithique supérieur dans le Sud-Ouest de la France, Cahier du Quaternaire, 6, CNRS, Paris, $453 \mathrm{p}$.

DELPECH F. (1987) - L'environnement animal des magdaléniens, in M. Otte dir., Le Magdalénien en Europe, Actes du colloque de Mayence, $X I^{e}$ congrès UISPP, ERAUL, t. 38, Liège, p. 5-30.

DELPECH F. (1999) - Biomasse d'Ongulés au Paléolithique et inférences sur la démographie, Paléo, t. 11, p. 19-42.

DELPECH F., LE GALL O. (1983) - La faune magdalénienne de la grotte des Églises (Ussat, Ariège), Bulletin de la Société préhistorique de l'Ariège, t. 36, p. 91-118.

DELPECH F., VILLA P. (1993) - Activités de chasse et de boucherie dans la grotte des Églises, in J. Desse J. et F. Audoin-Rouzeau dir., Exploitation des animaux sauvages à travers le temps, XIII ${ }^{e s}$ rencontres internationales d'Archéologie et d'Histoire d'Antibes, IV colloque international de l'Homme et de l'Animal, APDCA-CNRS, Antibes, p. 79-102.

DELPECH F., DONARD E., GILBERT A., GUADELLI J.-L., LE GALL O., JACQUIN A.-M., PAQUEREAU M.-M., PRAT F., TOURNEPICHE J.-F. (1983) - Contribution à la lecture des paléoclimats quaternaires d'après les données de la paléontologie en milieu continental. Quelques exemples de flore et de faune d'Ongulés pris dans le Pléistocène supérieur, Paléoclimats, Bulletin de l'Institut géologique du bassin d'Aquitaine, Cahier du Quaternaire, 34, CNRS, Bordeaux, p. $165-177$.

EASTHAM A. (1998) - Magdalenians and snowy-owls: bones recovered at the grotte de Bourouilla (Arancou, Pyrénées-Atlantiques), Paléo, t. 10, p. $95-107$.

ENLOE J.G. (1999) - Hunting specialization: single-species focus and human adaptation, in J.-P. Brugal, F. David, J.G. Enloe et J. Jaubert dir. Le Bison : gibier et moyen de subsistance des hommes du Paléolithique aux Paléoindiens des grandes plaines, Actes du colloque international, Toulouse, 1995, APCDA-CNRS, Antibes, p. 501509.

FONTANA L. (1998a) - Mobilité et subsistance au Magdalénien dans le Languedoc occidental et le Roussillon, thèse de doctorat, université de Paris I, Paris, 2 t., 285 p.

FONTANA L. (1998b) - Subsistance et territoire au Magdalénien supérieur dans les Pyrénées : l'apport des données archéozoologiques de la grotte de Belvis, Préhistoire du sud-Ouest, Nouvelles Études, t. 5 , p. $131-146$

FONTANA L. (2003a) - Characterization and exploitation of the arctic hare (Lepus timidus) during the Magdalenian: surprising data from Gazel Cave (Aude, France)?, in S. Costamagno et V. Laroulandie dir., Mode de vie au Magdalénien : les apports de l'archéozoologie, Actes du XIV congrès UISPP, Liège, septembre 2001, BAR International Series, 1144, Oxford, p. 101-118.

FONTANA L. (2003b) - Le renne, l'aurochs et les volcans. Hommes, gibiers et reconquête forestière en Grande Limagne entre 12300 BP et 7500 BP, Paléo, t. 15, p. 87-104.

FOSSE P. (1999) - La grande faune mammalienne : remarques préliminaires, in C. Chauchat dir., L'habitat magdalénien de la grotte du Bourouilla à Arancou (Pyrénées-Atlantiques), Gallia Préhistoire, t. 41, p. $98-113$.

GEDDES D., CARRÈRE I., ROBERT M. (1985) - La Balma Margineda Animaux sauvages et animaux domestiques, Les dossiers Histoire et Archéologie, t. 96, p. 28-30.

GEDDES D., GUILAINE, J., COULAROU J., LE GALL O., MARTZLUFF M. (1990) - Postglacial environments, settlement and subsistence in the Pyrénées: the Balma Margineda, Andorre, in C. Bonsall dir., Mesolithic in Europe, Edinburg, p. 561-571.

GRAYSON D.K. (1984) - Quantitative zooarchaeology. Topics in the analysis of archaeological faunas, Academic Press, New York, 202 p.

GRIGGO C. (1996) - Établissement de courbes climatiques quantifiées à partir des communautés animales pléistocènes suivi d'une application aux gisements de l'abri Suard (Charente) et de la grotte de Bois Ragot (Vienne), Paléo, t. 8, p. 81-97.

HAWS J.A., HOCKETT B.S. (2004) - Theoretical perspectives on the dietary role of small animals in human evolution, in J.-P. Brugal et J. Desse dir., Petits animaux et sociétés humaines, XXIV rencontres internationales d'Archéologie et d'Histoire d'Antibes, octobre 2003 , APDCA-CNRS, Antibes, p. 533-546.

HEINZ C., BARBAZA M. (1998) - Environmental changes during the Late Glacial and Post-Glacial in the central Pyrenees (France): new charcoal analysis and archaeological data, Review of Palaeobotany and Palynology, vol. 104, p. 1-17.

HELMER D., MONCHOT H. (2006) - Un site mésolithique de chasse à l'aurochs (la Montagne, Sénas, Bouches-du-Rhône), Anthropozoologica, vol. 41, p. 215-228.

HOLLIDAY T.W. (1998) - The ecological context of trapping among recent hunter-gatherers: implications for subsistence in terminal Pleistocene Europe, Current Anthropology, vol. 39, p. 711-720.

JALUT G., MONSERRAT MARTI J., FONTUGNE M., DELIBRIAS G., VILAPANA J. M., JULIA R. (1992) - Glacial to Interglacial vegetation changes in the northern and southern Pyrénées: de-glaciation, vegetation cover and chronology, Quaternary Science Reviews, vol. 11 , p. $449-480$

JALUT G., GALOP D., BELET J.M AUBERT S ESTEBAN AMAT A., BOUCHETTE A., DEDOURAT J.-J., FONTUGNE M. (1998) - Histoire des forêts du versant nord des Pyrénées au cours des 30000 dernières années, Journal de Botanique de la Société botanique de France, t. 5, p. 73-84.

LACOMBE S. (1998) - Préhistoire des groupes culturels au Tardiglaciaire dans les Pyrénées centrales, thèse d'université, université Toulouse-Le Mirail, Toulouse, 385 p. 
LAROULANDIE V. (2000) - Taphonomie et archéozoologie des oiseaux en grotte: applications aux sites paléolithiques du Bois Rago (Vienne), de Combe Saunière (Dordogne) et de la Vache (Ariège), thèse d'université, université de Bordeaux I, Bordeaux, 396 p.

LAROULANDIE V. (2004) - Exploitation des ressources aviaires durant le Paléolithique en France : bilan critique et perspectives, in J.-P. Brugal et J. Desse dir., Petits animaux et sociétés humaines, $X X I V^{\text {es }}$ rencontres internationales d'Archéologie et d'Histoire d'Antibes, octobre 2003, APDCA-CNRS, Antibes, p. 163-172.

LAROULANDIE V. (2005) - Bird Exploitation Pattern: the Case of Ptarmigan Lagopus sp. in the Upper Magdalenian Site of La Vache (Ariège, France), in G. Grupe et J. Peters dir., Feathers, grit and symbolism. Birds and humans in the ancient Old and New Worlds, Proceedings of the 5th Meeting of the ICAZ Bird Working Group, Munich, 26-28 July 2004, Documenta Archaeobiologiae, ${ }^{\circ} 3$, Rahden/ Westf. : Verlag Marie Leidorf, p. 165-178.

LE GALL O. (1993) - Évolution des pêches de l'Épipaléolithique au Néolithique ancien, Préhistoire Anthropologie méditerranéennes, t. 2 , p. $135-142$.

LE GALL O. (2003) - Des Magdaléniens et... des poissons, in S. Costamagno et V. Laroulandie dir., Mode de vie au Magdalénien: les apports de l'archéozoologie, Actes du XIV congrès UISPP, Liège, septembre 2001, BAR International Series, 1144, Oxford, p. 119128.

LUBELL D. (2004) - Prehistoric edible land snails in the circumMediterranean: the archaeological evidence, in J.-P. Brugal et J. Desse dir., Petits animaux et sociétés humaines, XXIV res rencontres internationales d'Archéologie et d'Histoire d'Antibes, octobre 2003, APDCACNRS, Antibes, p. 77-98.

MARTIN H. (1994) - Nouveaux milieux, nouveaux chasseurs, une approche des comportements au post-Glaciaire à travers l'étude des saisons de capture du gibier, thèse de doctorat, université de Toulouse 2-Le Mirail, Toulouse, 383 p.

MOIGNE A.-M., BAILLS H., GRÉGOIRE S. (1998) - Les Magdaléniens de la grotte des Conques (Pyrénées-Orientales), caractérisation du site d'après les restes osseux et l'outillage, in J.-P. Brugal, L. Meignen et M. Patou-Mathis dir., Économie préhistorique : les comportements de subsistance au Paléolithique, XVIII ${ }^{\text {es }}$ rencontres internationales d'Archéologie et d'Histoire d'Antibes, APCDA-CNRS, Antibes, p. 397-411.

MÜLLER W. (2004) - One horse or a hundred hares? Small game exploitation in an Upper Palaeolithic context, in J.-P. Brugal et J. Desse dir., Petits animaux et sociétés humaines, XXIV ${ }^{\text {s }}$ rencontres internationales d'Archéologie et d'Histoire d'Antibes, octobre 2003, APDCACNRS, Antibes, p. 489-498.

OMNÈS J. (1982) - La grotte ornée de Labastide (Hautes-Pyrénées), J. Omnès, Lourdes, 352 p.

PAILHAUGUE N. (1995) - La faune de la salle Monique, grotte de la Vache (Alliat, Ariège), Bulletin de la Société préhistorique de l'Ariège t. L, p. 225-289.

PASTY J.-F., ALIX P., BALLUT C., GRIGGO C., MURAT R. (2002) - Le gisement épipaléolithique à pointes de Malaurie de Champ-Chalatras (Les Martres-d'Artière, Puy-de-Dôme), Paléo, t. 14, p. 101-176.

PATOU M. (1984) - La faune de la galerie rive droite du Mas d'Azil (Ariège) : données paléoclimatiques et palethnographiques, Bulletin de la Société préhistorique française, t. 81, p. 311-319.

PATOU M. (1985) - Dénombrement et détermination de l'âge des rennes (Rangifer tarandus) provenant de la galerie rive droite du Mas d'Azil (Ariège) : méthodes et résultats, Revue de Paléobiologie, t. 4, p. 7178.

PATOU-MATHIS M., BOUKHIMA H. (1996) - Les grands mammifères des grottes des Hyènes et du Pape à Brassempouy (Landes) : résultats préliminaires, Actes du $118^{e}$ congrès national des Sociétés historiques et scientifiques, Pau, 1993, CTHS, Paris, p. 457-472.

PIGEOT N. (1987) - Magdaléniens d'Étiolles. Économie de débitage et organisation sociale (L'unité d'habitation U5), XXV' suppl. à Gallia Préhistoire, $160 \mathrm{p}$.
REIMER P.J., BAILLIE M.G.L., BARD É,, BAYLISS A., BECK J.W., BERTRAND C., BLACKWELL P.G., BUCK G., BURR C.E., CUTLER K.B., DAMON P.E., EDWARDS R.L., FAIRBANKS R.G., FRIEDRICH M., GUILDERSON T.P., HUGHEN K.A., KROMER B., McCORMAC F.G., MANNING S., BRONK RAMSEY C., REIMER R.W., REMMELE S., SOUTHON J.R., STUIVER M., TALAMO S, TAYLOR F.W, VAN DER PLICHT J et WEYHENMEYER CE. (2004) - IntCal04 Atmospheric radiocarbon age calibration, 26-0 ka BP, Radiocarbon, vol. 46, p. 1029-1058.

ROZOY J.-G. (1992) - Le propulseur et l'arc chez les chasseurs préhistoriques. Techniques et démographies comparées, Paléo, t. 4, p. 175 193.

ROZOY J.-G. (1978) - Les derniers chasseurs, Bulletin de la Société archéologique Champenoise ( ${ }^{\circ}$ spécial juin), 3 tomes, 1256 p., 294 fig., 259 pl., 80 tabl.

ROWLEY-CONWY P. (1986) - Between cave painters and crop planters: aspects of the temperate European Mesolithic, in M. Zvelebil dir., Hunters in transition. Mesolithic societies of temperate Eurasia and their transition to farming, Cambridge University Press, Cambridge, p. 13-32.

SACCHI D. (2003) - Le Magdalénien apogée de l'art quaternaire, La Maison des Roches, Paris, 126 p.

SÉRONIE-VIVIEN M.-R. (1994) - Données sur le Lapin azilien. Le matériel du gisement de Pégourié, Caniac-du-Causse (Lot), Bulletin de la Société préhistorique française, t. 91, p. 378-384.

SÉRONIE-VIVIEN M.-R. (2002) - Évidence de transport sud/nord de matières premières siliceuses en Aquitaine, in N . Cazals dir., Rapport de PCR, Comportements techniques et économiques des sociétés du Paléolithique supérieur en contexte pyrénéen, service régional de l'Archéologie de Midi-Pyrénées, p. 64-65.

SIMONNET R. (1982) - Grandes lames de silex dans le Paléolithique supérieur des Pyrénées centrales, Bulletin de la Société préhistorique de l'Ariège, t. 37, p. 61-105.

SIMONNET R. (1998) - Le silex et la fin du Paléolithique supérieur dans le bassin de Tarascon-sur-Ariège, Bulletin de la Société préhistorique Ariège Pyrénées, t. 53, p. 181-222.

SIMONNET R. (1999) - De la géologie à la Préhistoire. Le silex des pré-Pyrénées. Résultats et réflexions sur les perspectives et les limites de l'étude des matières premières lithiques, Paléo, t. 11, p. 71-88.

STINER M.C. (2001) - Thirty years on the «broad spectrum revolution» and Palaeolithic demography, Proceedings of the National Academy of Sciences, vol. 98, p. 6993-6996.

STINER M.C., MUNRO N.D., SUROVELL T.A., TCHERNOV E., BAR-YOSEF O. (1999) - Palaeolithic population growth pulses evidenced by small animal exploitation, Science, vol. 283, p. 190194.

TARRIÑO A. (2001) - El sílex en la Cuenca Vasco Cantábrica y Pirineo navarro : caracterización y su aprovechamiento en la prehistoria, Tesis Doctoral, Universidad del País Vasco, 364 p.

TURQ A., DETRAIN L., VIGIER S. (2000) - L'importance de l'accessibilité des gîtes dans l'étude de l'approvisionnement en matières premières lithiques : 1'exemple du Haut-Agenais (Lot-et-Garonne), Les derniers chasseurs-cueilleurs d'Europe occidentale (13000-5500 av. J.-C.), Actes du colloque international de Besancon, octobre 1998 Presses universitaires franc-comtoises, Besançon, p. 267-276.

VALDEYRON N. (1994) - Le Sauveterrien : cultures et sociétés mésolithiques dans la France du sud durant le $X^{e}$ et le $I X^{e}$ millénaire BP. mémoire de doctorat nouveau régime, UTM, Toulouse, 2 vol., 584 p., 141 fig., 1 carte h. t.

VALDEYRON N. (2000) - Géographie culturelle du Mésolithique récent/final dans le Sud-Ouest de la France, in M. Leduc, N. Valdeyron et J. Vaquer dir., Sociétés et espaces, Rencontres méridionales de Préhistoire récente, $3^{e}$ session, Toulouse, novembre 1998, Archives d'Écologie préhistorique, Toulouse, p. 23-34.

VALENTIN B. (2005) - Transformations de l'industrie lithique pendant l'Azilien. Étude des niveaux 3 et 4 du Bois Ragot, in A. Chollet et 
V. Dujardin dir., La grotte du Bois Ragot à Gouex (Vienne). Magdalénien et Azilien, Mémoire de Société préhistorique française, t. XXXVIII, Paris, p. 89-182.

VILETTE P. (1999) - Bilan provisoire sur la chasse aux oiseaux pendant le Leptolithique dans le Sud de la France, in D. Sacchi dir., Les faciès leptolithiques du nord-ouest méditerranéen : milieux naturels et culturels, Actes du XXIV congrès préhistorique de France, Carcassonne. septembre 1994, Société préhistorique française, Paris, p. 267-276.

WINTERHALDER B.P. (1981) - Optimal foraging strategies and huntergatherer research in anthropology: theory and models, in B.P. Winterhalder et E.A. Smith dir., Hunter-gatherer foraging strategies: ethnographic and archaeological analyses, University of Chicago Press, Chicago, p. 13-36.

\section{David COCHARD}

Jean-Georges FERRIÉ Véronique LAROULANDIE UMR 5199 du CNRS-PACEA Université Bordeaux I Avenue des Facultés, 33405 Talence coch@club.internet.fr jg.ferrie@ipgq.u-bordeaux1.fr v.laroulandie@ipgq.u-bordeaux1.fr

Nathalie CAZALS

UMR 7041 du CNRS-Arscan Maison René Ginouvès 21, allée de l'Université 92023 Nanterre Cedex cazals.nathalie@free.fr

athieu LANGLAIS

Nicolas VALDEYRON

Michel BARBAZA

Morgane DACHARY Hélène MARTIN

UMR 5608 du CNRS-TRACES

Maison de la Recherche

Université Toulouse-Le Mirail

5, allées Antonio-Machado

31058 Toulouse Cedex 9

costamag@univ-tlse2.fr

langlais@univ-tlse2.fr

valdeyro@univ-tlse2.fr

barbaza@univ-tlse2.fr

Morgane.dachary@wanadoo.fr martinhelene2001@yahoo.fr
Didier GALOP

UMR 6565 du CNRS

Laboratoire de Chrono-écologie Université de Franche-Comté, Besançon didier.galop@univ-fcomte.fr

Sylvie PHILIBERT

UMR $6130 \mathrm{du}$ CNRS-CEPAM 250, rue Albert-Einstein Sophia Antipolis, 06560 Valbonne 